\title{
The Importance of fungous diseases in immunology
}

\author{
Dr. J. Walter Wilson \\ Professor of Medicine, Dermatology, University of California
}

1. Success in discovering chemical and antibiotic antagonists against microbes has caused a reduction of effort in the study of immunology.

2. There are many examples of microbes which by mutation have produced and perpetuated strains resistant to those therapeutic agents, with which they have been in contact. It seems likely that all can do so if allowed enough time and contact.

3. It is probable that all of the antibiotics furnished by nature have been already discovered or soon will be. Chemists may succeed in changing these molecules enough to confuse the microbes for a time, but eventually such changes will be great enough to result in the loss of all antibiotic activity by these molecules.

4. For the above reasons, I believe that the presently successful antibiotic era will last but a few decades, certainly less than a century, which is a very short time in relation to the life of the human race.

5. It is admitted that there is no such definite limitation on the discovery of antimicrobial chemicals other than those furnished by nature's forms of life. However, as in the past, it is diffcult to avoid serious toxicity to human beings in certain compounds that are toxic to microbes.

6. I conclude that we should now redouble our efforts in studying immunology while we still have a "breathing spell" furnished by our presently effective therapeutic agents, to be ready when they no longer work well.

7. Most of the past study of immunology has been directed toward the great scourges of mankind, such as tuberculosis, leprosy, syphilis, cholera and plague. There have been developed effective methods of protecting normal human beings by vaccination against a subsequent contact by some of these microbes, but not against others.

8. We have been only slightly successful in discovering methods of assisting a person who already possesses an infection by any immunologic means. This sort of knowledge is what we need most of all.

9. I believe that certain fungous diseases present a pattern of immunologic resistance which is much less complicated than that in tuberculosis or leprosy, which remain unconquered. For example, I think that studying histoplasmosis and coccidioidomycosis intensively could furnish us with clues which could then be used to enable us to find our way to success in more complicated diseases.

10. Until recently it was believed that the systemic fungous diseases occurred but very raiely because the causative fungi were only rarely contacted. Now it is known that the reverse is true, that is that (particularly in some geographic areas) almost every person actually acquires the disease, but in a form so mild as to usually remain unrecognized, but nevertheless followed by the development of a complete and long-lasting specific immunity against reinfection. Only an occasional person becomes seriously involved and may die, and these possess some defect in their mechanisms of specific immunologic resistance. Thus it is now known that 30,000,000 persons have had histoplasmosis with only about 300 deaths. $10,000,000$ people have had coccidioidomycosis, with perhaps 2000 deaths. Such mechanisms are almost perfect and are therefore worthy of more study.

11. We should study intensively those persons who do not resist these disease in the manner of 
the overwhelming percentage of "normal" individuals, hoping thereby to find where they deviate from the normal, and thus to be able to help them to correct their defects and become normal. If we can succeed in one such disease, the methods may be then applied to other more prevalent diseases.

12, The deep fungous infections, particularly histoplasmosis and coccidioidomycosis, have much to offer as tools in the study of immunology. They offer a series of specific tests, serologic and intracutaneous, which seem to be able to reveal the level of the patient's ability to resist the disease immunologically, as well as the amount of the disease which is present for him to fight. These diseases can produce as many variations in clinical pictures as do tuberculosis or leprosy, and yet they seem to behave more simply from the immunologic standpoint.

13. Histoplasmosis and coccidioidomycosis indicate that it is the polysaccharide content of the causative organisms which is important in immunologic resistance, and not the proteins heretofore emphasized in tuberculosis. Recently polysaccharides have been found to be equally important in tuberculosis.

14. It is important to realize that animal experimentation of the usual type cannot answer these questions, because the animals used are normal. It is only by first studying the abnormal human beings (who because of their abnormality develop these diseases in serious form) that we can understand their important defects. Then perhaps similar defects could be produced in a group of animals, which could be subsequently infected and studied.

15. Clinicians can and must enter into these investigations, by making sure that all such rare cases are adequately studied and the results recorded in detail, thus eventually furnishing an "experimental" group which cannot be duplicated in animals at present. 